\title{
ONLINE REUSABLE GOODS MANAGEMENT SYSTEM (ORGMS)
}

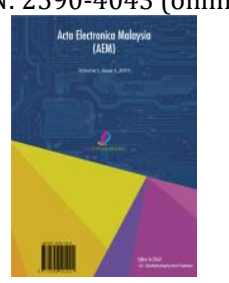

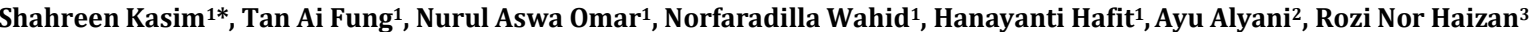 \\ ${ }^{1}$ Soft Computing and Data Mining Centre, Faculty of Computer Science and Information Technology, Universiti Tun Hussein Onn, Johor, Malaysia \\ ${ }^{2}$ Findbulous Technology Sdn Bhd, Batu Pahat, Johor \\ ${ }^{3}$ Faculty of Computer Science and Information Technology, Universiti Putra Malaysia \\ *Corresponding author email: shahreen@uthm.edu.my
}

This is an open access article distributed under the Creative Commons Attribution License, which permits unrestricted use, distribution, and reproduction in any medium, provided the original work is properly cited.

\section{ARTICLE DETAILS}

Article History:

Received 3 July 2017

Accepted 3 October 2017

Available online 9 November 2017

Keywords:

Online Reusable Goods

Management System, web engineering, life-cycle, C\#.NET.

\section{ABSTRACT}

If we do not know how to manage the unwanted items, it is quite a big problem. In Malaysia, we have produced abou thousands of tonnes of waste everyday (Md. Abdul Jalil, 2010). The best solution for this problem is recycling. Actually, the things that we throw can almost be reused. Therefore, Online Reusable Goods Management System (ORGMS) is a web-based system that will give suggestions on how to manage unwanted items. This system is an online community bulletin board where individuals can offer free, selling items or browse through listings and find a treasure of their own. In addition, this system also provides many Do It Yourself (DIY) methods to manage their reusable items in creative and useful ways. Furthermore, when users are posting listing, they are advised to donate money to certain charity houses or community. For methodology, web engineering is used in this project. Web engineering enables to guide this project through developing and implementing activities and tasks in well-defined phases of the project implementation life-cycle. In addition, this system is developed by using C\#.NET as programming language and Microsoft SQL server as database server. As a conclusion, this system provides users a great way to recycle and help out the community.

\section{INTRODUCTION}

If we do not know how to manage the unwanted items, it is quite a big problem. Therefore, Online Reusable Goods Management System is a system that will be developed to give some suggestions on how to manage unwanted items. This will make users to reuse their old items rather than throwing them into the trash [1]. In addition, this will also help to reduce disposal needs and costs. With the system, users can find the different and useful ways to manage their unwanted items by choosing what reusable items that they have from a list of categories of items. The category of items is divided by two, they are smaller items and larger items. The category of larger items includes furniture, books, electronics appliances and others. For the category of smaller items includes papers, cans, clothes, plastics, bottle and others. This system will also sort out the most appropriate methods to manage the reusable items especially for the smaller items, the methods are called Do It Yourself (DIY) methods [2]. Besides that, users must sign up as member first so that they have a chance to give their comment and feedback and to post their listings. Moreover, users can post listings that users wish to get or browse for. Each listing contains a description of the items along with a name, email 2 address, and any cost. The actual exchange transactions are carried out directly between the interested parties. Furthermore, when users are posting listing to sell their items, they are advised to donate money to certain charity house and community which is choosing by their own. This system will always update, add and delete the listings and DIY methods. As a conclusion, this system provides users a great way to recycle and help out the community.

\section{PROBLEM STATEMENT}

When we are going to clean up the house, we will find a lot of items that we no longer want or need. For example, kids toy, books, kitchen gadgets and electronic goods. Actually, these types of waste and unwanted items can be reused and recycled. However, we do not know how to manage them or contact with agent to collect them [3]. We are also lazy to send to recycle center, so we just simply throw them away and go to landfill. It is become easy for us to adopt an 'out of sight, out of mind' attitude, without any thought to the consequences. This is damaging for the environment. Once things get into landfill, they either stay there for hundreds or thousands of years. Taking things to the landfill is not a good option for us.
On the other hand, there are many people tightening their belts and cannot afford to buy new items. For example, especially the students will search for basic kitchen equipment and electronic appliances that many people throw out regularly [4]. As a result, there is a need to have this system to browse, give away or sell the reusable items to create a less waste community. This system is an online community bulletin board where individuals can offer free, selling items or browse through listings and find a treasure of their own. It is free to join, free to post and free to browse.

In addition, for the smaller items like papers, cans, clothes; this system also provides DIY methods to manage these reusable items in creative and useful ways. Furthermore, who is using this system also advised to contribute or donate to charity houses or community. Thus, ORGMS surely will help users in managing, getting their reusable items with the best ways and also can help out the community.

\section{SURVEY ON EXISTING SYSTEMS}

Survey on other existing systems in the market is important for the development of a system. It allows the advantages of that certain systems to be used as guidelines of the development itself. Among the systems surveyed are
i. 2GOOD2TOSS.com;
ii. SnaffleUp.co.uk;
iii. Lelong.com.my.

\subsection{G00D2TOSS.com (http://www.2good2toss.com)}

2good2toss.com is Washington's online exchange for reusable building materials and household items. The objective of the site is to facilitate the recycling and repurposing of materials and items that would otherwise be disposed at Washington state landfills and waste to energy facilities, in addition to promoting an important environmental ethic. Users can post listings of items and materials that they wish to get rid of or browse for those currently available in their area. Each listing contains a description of the materials along with a name and telephone number and any cost or delivery information. When users find an interesting item, users can contact the user that posted it, and make a pick up or delivery arrangement. The actual exchange transactions are carried out directly 
between the interested parties. 2good2toss.com listings include antiques and collectibles, auto parts, books and clothing. In addition, computers, camping gear and electrical supplies are available.

\subsection{SnaffleUp.co.uk (http://www.snaffleup.co.uk)}

SnaffleUp.co.uk is a service that promotes the recycling of unwanted but still useful items. SnaffleUp.co.uk makes a simple for users to donate the stuff that is cluttering up their home rather than throw it away. This makes a small but crucial difference to the environment by reducing the growing problem of landfill.

The SnaffleUp website also makes it simple for users to find useful items in their area for free. Search for a particular item in their area or browse what is on offer by category. Users can get emails when something they are on the lookout for becomes available. Not only do users get free stuff, users are also going to help environmental waste by recycling secondhand items.

In SnaffleUp, users can add pictures to their listings and they can find out instantly how far away an item is from their own home. Users will find the top 10 items closest to their postcode on the SnaffleUp site, so they will see in an instant what is around. Besides that, click on any item and a Google map shows users where to go to collect their item. Activities in SnaffleUp includes furniture reuse, IT reuse or recycling, office recycling, textile reuse or recycling, waste minimization and others.

\subsection{Lelong.com.my (http://www.lelong.com.my)}

Styles and functioning of Lelong.com.my similar to the international auction site eBay.com. Lelong.com.my powers a robust and secure avenue for trading in Malaysia. The idea of an auction site in Malaysia has taken off with good response. With a local flavor, members feel a sense of belonging and constantly keep in contact with the Lelong Development Team to give feedback. Actually, Lelong.com.my was starting based on Consumer-to-Consumer (C2C) transactions, but for now moving to becoming a channel for Business-to-Consumer (B2C) relationships. Many distributors have realized the power of using the auction system and are now using this site to trade and grow their business.

The attractions of using Lelong.com.my is many of us have lots of items lying around the house that are not currently in use; whether second hand or new items that we have not gotten around to disposing of. This is a major factor that has brought many users to Lelong.com.my. The track record for selling items is extremely good. Approximately $70 \%$ of all items posted for auction on Lelong.com.my are sold. Hence, users stand a good chance of obtaining a successful transaction. The interface is very userfriendly and easy to use. The styles and functions of Lelong.com.my are similar to the international auction site such as eBay.com. Lelong.com.my provides many products and services to the public such as clothing and accessories, books, comics, watches, pens, clocks and others with the entire picture. Besides, Lelong.com.my also provides the search engine to easy the consumer to search their needs faster with the key words. Problems that arise can also be posted in the forum and many users play a major role in replying and helping out others. With all our on-going efforts, a community of auction users has formed which are extremely diverse, and who actively interact amongst one another. This causes them to feel at home and comfortable trading with other members.

A comparison between the existing systems and proposed system are done, according to several features. In this table shows that only Lelong.my needs to pay the posting fee. However, posting fee is not needed by the other three systems which are 2GOOD2TOSS, SnaffleUp and ORGMS. Besides, this table also shows that all systems can let user to rate and give feedback except the 2GOOD2TOSS. Moreover, all system provides the quick search tool so that users can find their match items quickly and easily. Furthermore, in this comparison shows that 2GOOD2TOSS, SnaffleUp and ORGMS are more focus on recycle. Then, only Lelong.my is more focus on business purpose. In addition, for ORGMS will also focus on the charity purpose. On the other hand, there are many advertisements in the front page of Lelong.my. This will take longer time to download that page. The other three systems do not have this problem. Finally, there is only ORGMS will provide DIY methods which will teach users how to use reusable items to do DIY crafts.

\section{DESIGN AND IMPLEMENTATION}

The deliverable of the implementation phase is the system prototype of ORGMS that demonstrates the system defined in the design phase based on the functions and features specified in the analysis phase. This section explains the flow and execution of ORGMS with some sample data.
However, only the core functions and features will be demonstrated and displayed in figures here.

Search Items Page has divided into three sections, there are advance search, quick search and all items. As shown in below Figure 1. For advance search items, users can search by keyword and category, or only search by category. The SQL command is select all from Items table where Category gets from the Dropdownlist; and keyword taken from textbox must inside the ItemTitle and Location.

Moreover, SQL command for quick search items is select all from Items table where State gets from Dropdownlist1; and ItemType gets from the Dropdownlist2. As shown in Figure 2. For all items, users can also search by keyword. SQL command used is select all from Items table where keyword gets from the textbox is inside the ItemTitle or ItemDescription or Location. As shown in Figure 3.

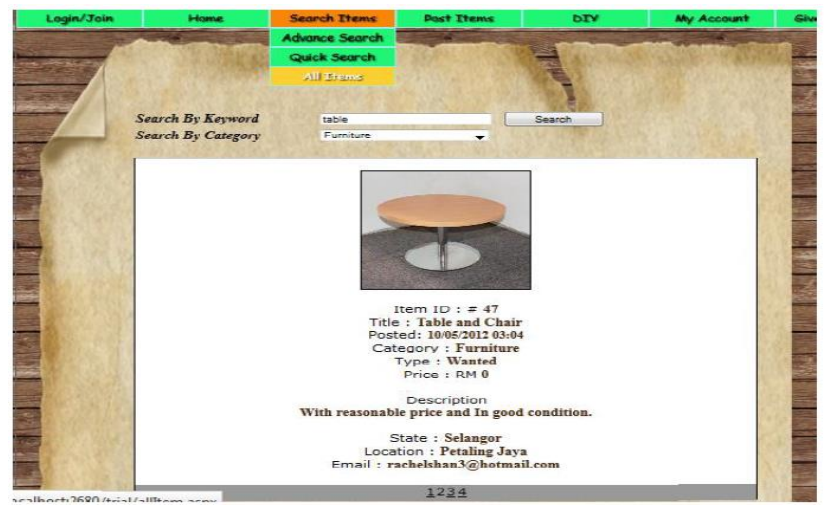

Figure 1: Interface of Advance Search Items Page

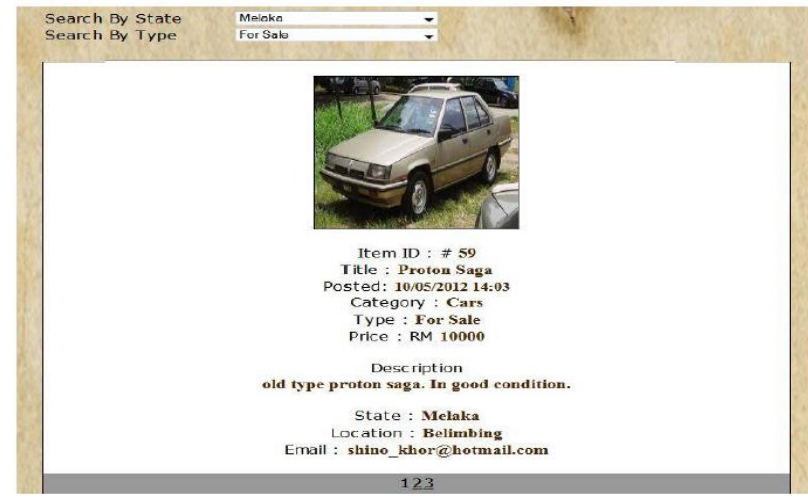

Figure 2: Interface of Quick Search Items Page

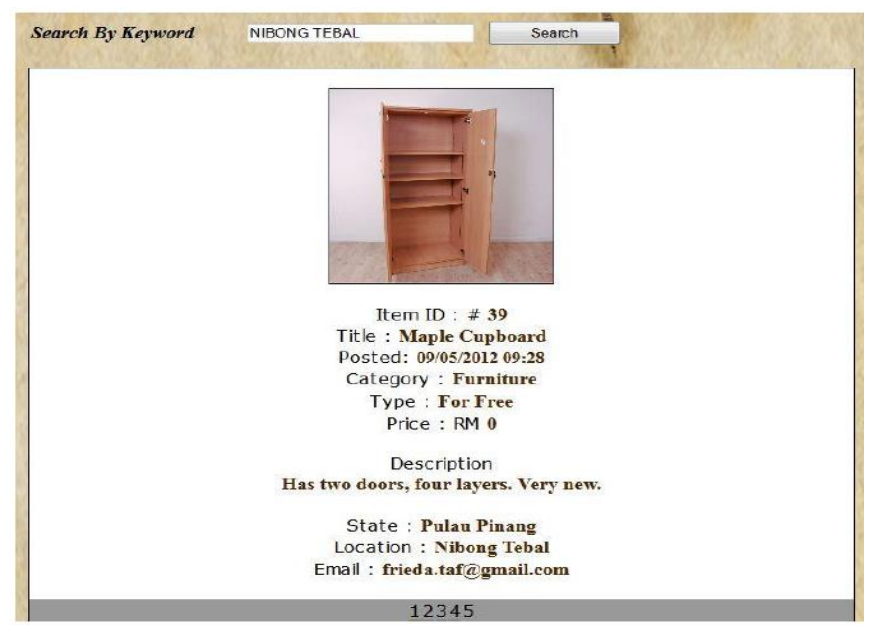

Figure 3: Interface of All Items Page

Search DIY Page has divided into two sections, there are advance search and all DIY. As shown in below Figure 4. For advance search DIY, users can search by keyword and category, or only search by category. The SQL command is select all from diy table where diyCat gets from the Dropdownlist; and keyword taken from textbox must inside the diyTitle and material.

Moreover, SQL command for all items, users can also search by keyword 
SQL command used is select all from diy table where keyword gets from the textbox is inside the diyTitle or material. As shown in Figure 5.

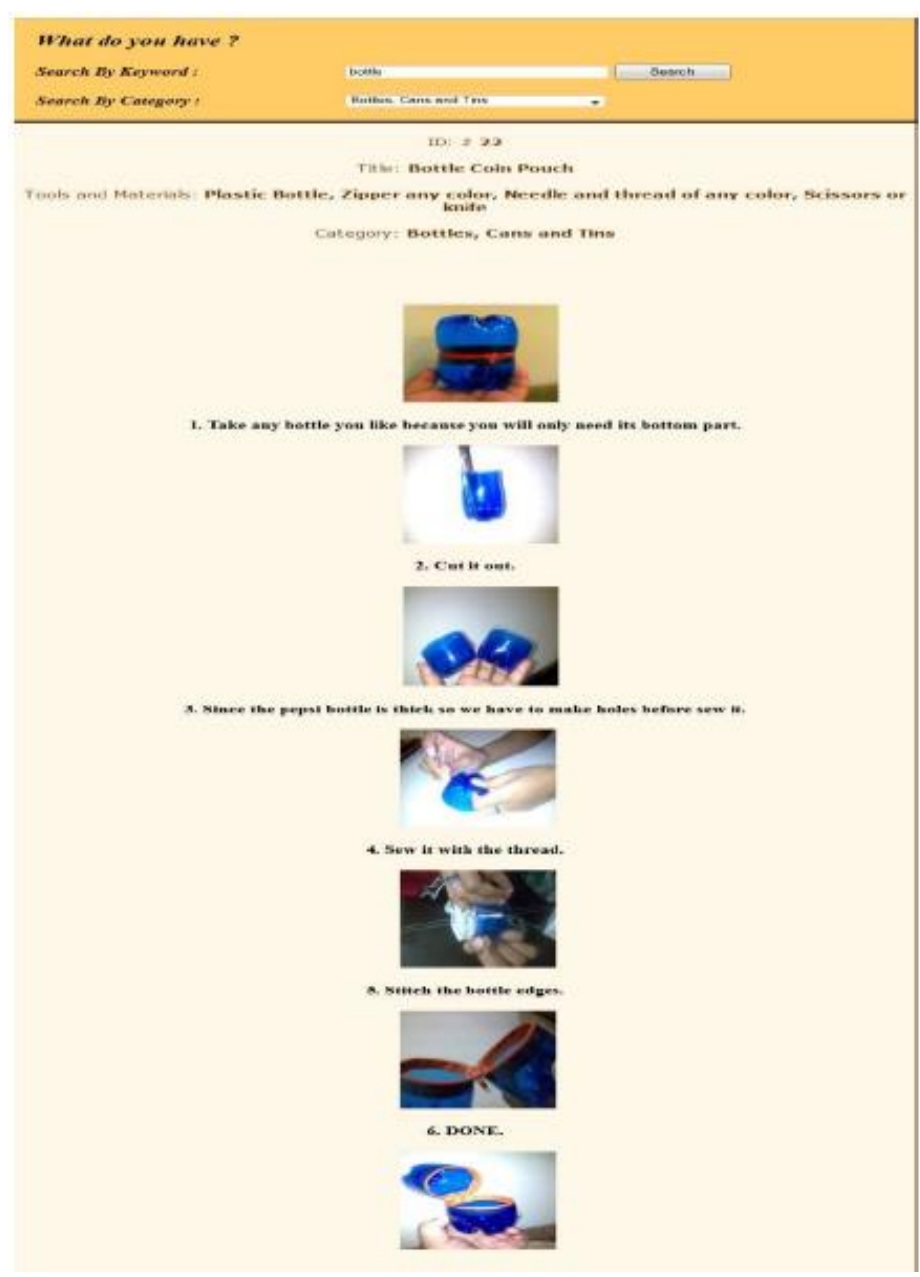

Figure 4: Interface of Advance Search DIY Page

\section{USER ACCEPTANCE TESTING}

User acceptance testing involves procedures for identifying acceptance criteria for interim life cycle products and for accepting them. To accomplish the testing, user evaluation form is prepared for the users to be filled-up during the system testing process. Please refer to Appendix C for the evaluation form. After the system is ready for implementation, this system also has been tested by twenty (20) real users to perform user acceptance testing. The overall result as shown in Figure 5.20 below. From the 20 respondents, there are five (5) respondents rate for very good which is $25 \%$. Then, eleven (11) respondents rate for good which is $55 \%$. Only four (4) respondents rate for medium which is $20 \%$. There is no respondent rate for weak and satisfactory.

\section{CONCLUSION}

The final conclusion for this project is that the proposed system has been successfully developed and fulfilled its objectives. Methodology of web engineering has been used in order to develop the proposed system. Five major phases which are business and requirements analysis, design, implementation, testing and evaluation and all UML diagrams are used to

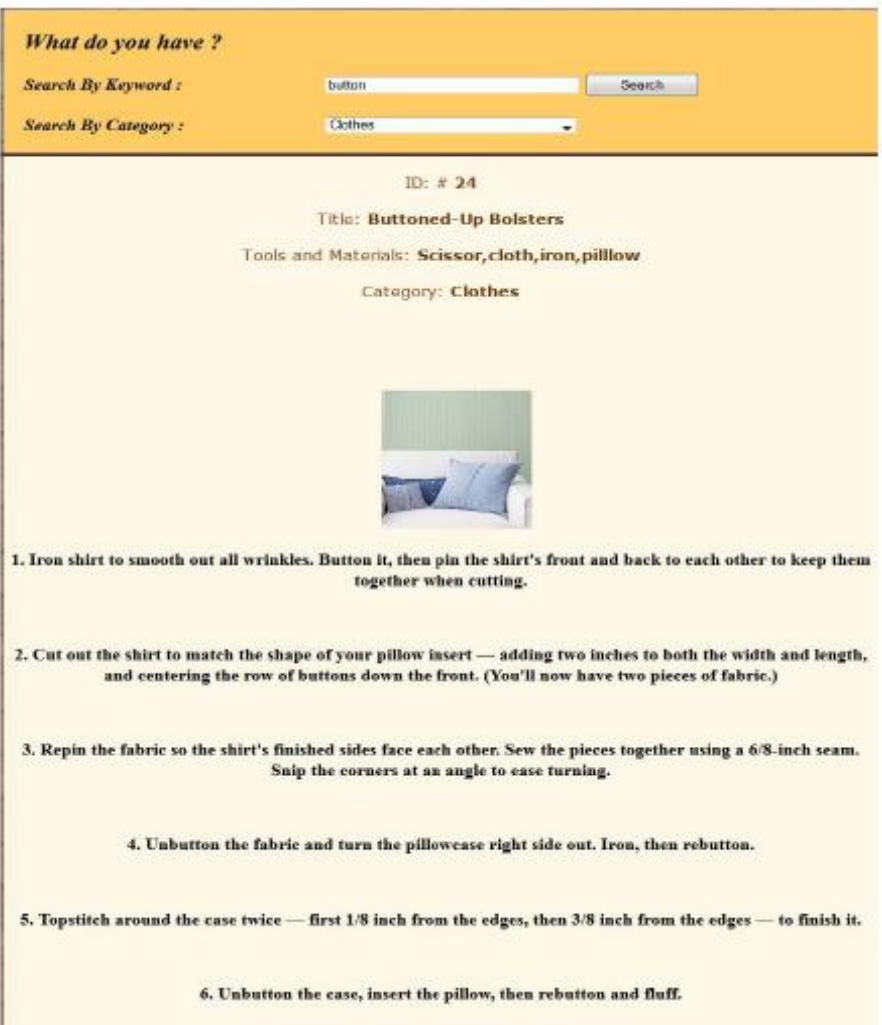

Figure 5: Interface of All DIY Page

serve as the guidance during implementation process. Based on the system modes, the system is implemented to an actual system. Although the proposed system has achieved its objectives within the predefined scope, several recommendations for future works can be done in future to enhance the system beyond the current limitation to be a more functional, more useful and more compatible system.

\section{REFERENCES}

[1] Turban, E., King, D. 2011. Electronic Commerce 2012. 7th Edition. United Kingdom. Pearson.

[2] Abebooks. 2015. A Guide to Used Goods Condition. Retrieved on May 2015 from www.abebooks.com

[3] Roth, R.M., Dennis, A., Wixom, B.H. 2013. Systems Analysis and Design. 5th Edition. United States. John Wiley \& Sons, Inc.

[4] Budd A. T. 2002. An Introduction to Object-Oriented Programming. 3rd Edition. New Jersey: Addison Wesley. Longman. 